Horizons philosophiques

\title{
Au sujet de la nouvelle traduction de la Bible
} Entretien avec Robert David.

\section{Maurice Burgevin}

Volume 13, numéro 1, automne 2002

Religion et pluralisme

URI : https://id.erudit.org/iderudit/801226ar

DOI : https://doi.org/10.7202/801226ar

Aller au sommaire du numéro

Éditeur(s)

Collège Édouard-Montpetit

ISSN

1181-9227 (imprimé)

1920-2954 (numérique)

Découvrir la revue

Citer cet article

Burgevin, M. (2002). Au sujet de la nouvelle traduction de la Bible : entretien avec Robert David. Horizons philosophiques, 13(1), 91-104.

https://doi.org/10.7202/801226ar d'utilisation que vous pouvez consulter en ligne.

https://apropos.erudit.org/fr/usagers/politique-dutilisation/ 


\section{AU SUJET \\ DE LA NOUVELLE \\ TRADUCTION DE LA BIBLE \\ ENTRETIEN \\ AVEC ROBERT DAVID}

Le numéro du 30 août au 5 septembre 2001 du très laïc Nouvel Observateur consacrait neuf pages à ce qui allait devenir un événement : la publication de la Bible, Nouvelle Traduction (la BNT) ${ }^{1}$. Ce travail avait associé, pendant près de sept ans, des biblistes francophones d'Europe et du Canada avec des écrivains croyants ou non-croyants (en binômes). Résultat : un monument littéraire, un texte décapé, libre et surprenant. Les livres fondateurs du christianisme (la Bible $\left.{ }^{2}\right), \tau \alpha \operatorname{Br} \beta \lambda_{l} \alpha$ sont proposés à l'attention de toute personne, croyante ou non, qui s'interroge sur les sources de la civilisation occidentale, ou plus simplement, sur l'existence humaine ou, plus simplement encore, à toute personne qui aime les beaux livres ${ }^{3}$.

\section{- Monsieur Robert David, pourquoi cette nouvelle traduction de la Bible?}

- L'une des raisons principales, c'est que les derniers travaux de traduction scientifique de la Bible, en français, dataient d'une trentaine d'années. C'était la TOB4, la Bible de Jérusalem et la Bible d'Osty, des traductions qui, à l'époque, s'inspiraient beaucoup des recherches effectuées en analyses historico-critiques ${ }^{5}$, et qui, dans l'ensemble, se ressemblent passablement au niveau de leur contenu (traduction, introductions, notes, annexes).

1. Montréal, Médiaspaul et Paris, Bayard, 2001.

2. Le mot «Bible» vient du grec $\tau \alpha \beta i \beta \lambda_{1} \alpha$ : les petits livres.

3. Voici la présentation que fait de ce travail l'un de ses artisans, Robert David, professeur d'exégèse biblique à la Faculté de théologie de l'Université de Montréal. Pour la BNT, il a travaillé à la traduction du livre de Josué avec Jean Echenoz, prix Goncourt 1999. L'entrevue a été réalisée par Maurice Burgevin.

4. Traduction CEcuménique de la Bible.

5. Les approches historico-critiques mettent de l'avant diverses techniques d'analyse des textes bibliques. Celles-ci permettent de les resituer dans leurs contextes d'émergence, que ce soit du point de vue de la composition littéraire, de l'attribution d'un milieu de vie ou de l'identification de contextes sociologiques, de l'histoire de la rédaction ou de la transmission. Elles s'intéressent à la question: d'où viennent les textes et comment ont-ils été transmis? 
- Le projet de la BNT était de travailler non plus à partir de perspectives strictement historiques (diachronie), mais en rapport avec les développements qui se sont faits en exégèse depuis une trentaine d'années, principalement dans le domaine des approches littéraires (synchronie). Nous voulions aborder le texte biblique sous son aspect proprement littéraire, en mettant à profit les sensibilités littéraires de quelques écrivains et écrivaines de notre époque.

Considérer les textes bibliques comme littérature, cela ne s'était pratiquement pas fait depuis le XVIIIe siècle. Nous voulions quelque chose de neuf, rajeunir la traduction, l'inscrire dans la contemporanéité. Les traductions antérieures restent très bonnes, mais elles sont plutôt scolaires et académiques. L'équipe éditoriale, et les gens contactés pour travailler au projet, ont estimé que les textes, dans leurs langues d'origine (hébreu, araméen, grec), avaient une beauté esthétique remarquable, et qu'il fallait pouvoir leur rendre cette beauté dans la langue de réception (français contemporain). Une bonne partie de ce travail a été guidée par cette préoccupation esthétique, celle-ci appuyée sur une solide expertise exégétique et théologique. Tout le reste a concouru à cela, dont cette particularité essentielle au projet : I'utilisation des binômes exégète-écrivain. Les questions littéraires, le souci esthétique, dans une fidélité aux langues originales, ont été nos préoccupations premières et constantes. Ceci se reflète d'ailleurs dans les notes, qui sont presque toutes d'ordre littéraire. II n'y presque pas de notes concernant les données de type historique, géographique ou archéologique. Les Bibles "classiques" en regorgent, nous n'avions pas intérêt à en ajouter.

La BNT visait un autre objectif, tout aussi important que le souci esthétique et littéraire, celui de dépoussiérer les textes au niveau des mots, des expressions, parfois même du contenu du texte lui-même. Par exemple, traduire le premier verset de Genèse par "dieu crée ciel et terre" plutôt que par le traditionnel "Dieu créa le ciel et la terre»6 a des implications importantes au niveau du contenu théologique. Ces textes anciens ont une très longue histoire de traduction,

6. À la traduction traditionnelle de la Bible de Jérusalem : «Au commencement, Dieu créa le ciel et la terre. Or la terre était vide et vague, les ténèbres couvraient l'abîme, un vent de Dieu tournoyait sur les eaux"; on peut opposer la traduction de la $B N T$, avec la syntaxe, l'orthographe, la ponctuation et la disposition suivantes :

Premiers

Dieu crée ciel et terre

terre vide solitude

noir au-dessus des fonds

souffle de dieu

mouvements au-dessus des eaux 
d'interprétation, de méditation. On a quelquefois l'impression qu'ils sont figés dans 2000 ans et plus de traditions religieuses. L'objectif poursuivi ne visait pas à mettre de côté la longue histoire de la tradition et de la réception, mais de les sortir d'un certain carcan théologique dans lequel ils étaient un peu embourbés.

\section{- Autre exemple, on lit "Joie de ceux qui sont à bout de souffle ${ }^{7}$...}

- «Joie" rend-t-il exactement le $\mu \alpha \kappa \alpha p t o \sigma ?$ «À bout de souffle» correspond-t-il parfaitement au substrat grec? Voilà des questions dont on pourrait débattre longuement. II est indéniable qu'il y a des choix de traduction qui ont été faits, comme pour toutes les autres traductions existantes. André Myre ${ }^{8}$ et Marie-Andrée Lamontagne, qui ont proposé cette traduction, ont fait ces choix, c'est clair. Ils voulaient présenter la force d'évocation du texte pour aujourd'hui. Ils ont considéré qu'il était plus parlant pour les gens d'aujourd'hui de traduire ainsi plutôt que de conserver les trop éthérés "heureux" ou "âme de pauvre». Pour tous les textes, les binômes ont cherché à retrouver les substrats des mots en grec, en hébreu ou en araméen, et à les laisser éclater pour le lecteur. II ne s'agissait pas de donner LE sens au lecteur, mais de lui donner l'occasion de faire du sens pendant la lecture. II faut reconnaître qu'on se trouve parfois déstabilisé par rapport à ce que l'on a l'habitude de lire. Cela oblige à se positionner, à réfléchir, à se poser des questions.

- Vous parlez de "souffle» et non d'«esprit". Mais dans les écoles primaires et secondaires du Québec, on se prépare à donner des cours de uformation spirituelle et animation communautaire". La notion de spiritualité, isolée d'un contexte religieux, a-t-elle une assise dans la Bible?

- Peut-on isoler la spiritualité de quelque notion religieuse? Depuis les Lumières on a créé une sorte de dichotomie entre l'univers religieux et le reste du réel. On ne fait pas cette distinction-là chez les anciens. C'est nous, avec nos catégories, qui cherchons à séparer le religieux du quotidien. Dans les sociétés qui ont donné naissance aux textes bibliques, le rapport au religieux est fondamental; pas forcément le religieux tel qu'on l'entend aujourd'hui dans certains milieux réactionnaires, le religieux "patenté", mais le rapport au sacré, au

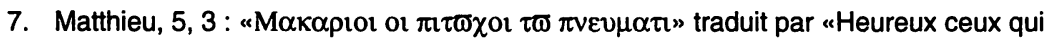
ont une âme de pauvre» (Osty, BJ), "Heureux les pauvres de cœur» (TOB). 
divin. Peut-on aborder ces textes-là en faisant fi du religieux? Je ne le pense pas. Ce sont d'abord et avant tout des textes religieux, qui témoignent de conceptions de Dieu, de rapports à l'absolu, dans toutes les sphères de la vie. Si on veut les aborder en faisant fi du religieux, on les écarte de leur essence.

Ceci dit, quelqu'un qui ne voudrait pas entretenir de rapport au religieux, dans le sens contemporain du terme, pourrait sans doute fréquenter ces textes pour ce qu'ils représentent d'héritage, témoins d'une réflexion située, d'une recherche de sens particulière, que l'on fait tous un jour ou l'autre. Est-ce que les réponses des anciens nous conviennent? Est-ce que leur rapport au réel est semblable au nôtre? Pas toujours. II en est même parfois passablement éloigné. Mais on ne peut nier qu'il y a dans ces textes le témoignage d'une véritable recherche de sens qui peut encore interpeller, questionner.

Un des objectifs de la BNT visait à rendre la Bible au monde contemporain, qui est moins "religieux" (dans le sens que ce mot avait dans les années '40, '50, '60), mais qui cherche quand même à retrouver ses racines. Qu'on le veuille ou non, une part importante de la culture occidentale est marquée par ces textes anciens. Si on se coupe de ces racines, on perd les assises permettant de comprendre et d'apprécier, à leurs justes valeurs, une partie importante des œuvres musicales, théâtrales, picturales et architecturales des siècles qui nous ont précédés, et même, de plusieurs œuvres contemporaines. On s'en rend compte à l'université : les étudiants qui n'ont pas eu accès à ces textes sont un peu dépourvus, parce qu'ils n'ont pas les fondements que procure cette culture. D'un strict point de vue culturel, ce sont des textes fondamentaux.

\section{- $Y$ avait-il des collaborateurs non-croyants sur le projet de la BNT?}

- II y avait des gens de divers horizons. Nous en avions un de la communauté juive ainsi que des personnes qui se déclaraient agnostiques. Toutefois, tous leurs témoignages concordent : le contact avec ces textes a été pour tous et toutes une expérience marquante. Les écrivains, même ceux qui se définissaient comme agnostiques, avaient d'ailleurs un rapport aux textes beaucoup plus "sacré" que celui que les exégètes pouvaient avoir, ce qui est peut-être normal considérant que nous, nous passons notre temps à

8. Bibliste, professeur honoraire à l'université de Montréal. 
travailler sur ces textes, quotidiennement. Chez les écrivains et écrivaines il y avait, par rapport aux textes, une espèce de distance, de vénération, d'intouchabilité, si vous permettez ce néologisme, que nous avons beaucoup moins quand nous fréquentons et analysons les textes d'un point de vue exégétique et scientifique (ce qui ne veut pas dire que nous n'en sentons pas aussi l'aspect sacré). Force est de le reconnaître, ces textes restent un monument essentiel, quelque chose qui vient nous chercher au plus profond de notre être. Nous avons été formés par ces textes et ils continuent d'occuper l'espace contemporain.

Aborder le texte biblique, le travailler, le rendre à nos contemporains, cela a été, pour certains et certaines, une expérience quasi mystique.

\section{- Pourrait-on avoir la même expérience en traduisant Homère?}

- Peut-être, mais je suis loin d'en être persuadé. Homère n'a pas le même impact que celui qu'ont eu, et ont encore, les textes bibliques. Tous les participants au projet ont dit qu'aborder le texte biblique, c'était aborder quelque chose de grand. Je ne suis pas sûr que l'on aurait abordé Homère de la même façon.

Je crois qu'une belle illustration du sérieux du travail et de son importance se trouve dans le fait que, chez les écrivains, personne n'a refusé la demande qui leur avait été formulée par le comité de direction de participer à ce vaste projet. C'était, au contraire, considéré comme un honneur, pas pour une gloire ou pour un plaisir personnels, mais parce qu'il y avait là un projet qui venait les chercher dans leur être profond. On ne s'attendait pas à cela.

\section{- Cela semble donner raison à Nietzsche qui écrivait que, dans l'Ancien Testament, on était en face de quelque chose de grand, de vénérables.}

- Nietzsche et d'autres ont bien senti cela. II y a des choses grandes, il y a des choses belles, il y a des grandeurs magnifiques. Je pense que ce travail qui a été fait par l'équipe pendant sept ans, a

9. "Dans l' "Ancien Testament" juif (...), il y a des hommes, des choses et des discours d'un si grand style que les textes grecs et hindous n'ont rien à leur opposer. On est stupéfait de crainte et de respect devant ces prodigieux vestiges de ce que fut jadis l'homme, (...) Le goût pour l'Ancien Testament une pierre de touche face à la "grandeur" et à la "petitesse"”. (Par-delà le bien et le mal \#52, Verviers, Marabout, 1975, p. 82-83). 
été soutenu parce qu'on considérait qu'il y avait des grandeurs dans ces textes et qu'on ne pouvait pas se permettre de laisser passer l'occasion de faire vivre un texte à travers une beauté exceptionnelle, rendue encore plus manifeste sous la plume de professionnels de l'écriture et des Écritures.

\section{- C'est presque paradoxal ce que vous dites. On est en train d'actualiser Tocqueville et on parle de la perte du sens de la noblesse.}

- Je ne suis pas sûr de la perte du sens de la noblesse (dans le sens noble (!) du terme). Cela dépend des milieux. Pour tous ceux et celles qui ont travaillé dans l'équipe, il y avait la volonté de faire en sorte qu'on aborde ces textes dans une perspective esthétique, dans leur beauté, je dirais presque dans leur corporéité. Alors qu'on l'a malheureusement trop souvent confiné dans une espèce de monde théologique et religieux éthéré et désincarné, nous voulions donner corps au texte. II y a un souffle dans le texte qui engage un corps à corps, un souffle à souffle, un être à être.

\section{- Quand on prend le début de la Genèse...10}

- On ne s'attend pas à lire ainsi ces versets. Je tiens à rappeler qu'il y a, à la base de chaque texte, le travail d'un exégète reconnu. On retient souvent de la $B N T$ le seul travail des écrivains et écrivaines. Ceci n'est pourtant qu'une fraction du long processus de traduction. Quand Frédéric Boyer traduit Genèse 1,1 de cette façon, il faut aussi penser au travail de l'exégète Jean L'Hour qui a approuvé cette traduction en dernière instance. Chaque exégète serait en mesure de défendre la traduction retenue au terme des échanges soutenus qui ont ponctué toute la démarche.

Quand j'ai lu Genèse 1,1 la première fois j'ai été surpris : qu'estce cela : "commencements" au pluriel? Que signifie cette traduction de beréshît? On avait "commencement", mais en écrivant "premiers (pluriel)», on ouvre de nouvelles options à la lecture. Non pas "Au commencement, Dieu créa» mais «Premiers Dieu crée ciel et terre". On passe au présent, et on met l'accent sur la primauté du ciel et de la terre plutôt que sur la temporalité d'un commencement qui nous échappe.

10. «Au commencement, Dieu créa le ciel et la terre.» (Bible de Jérusalem) «Premiers Dieu crée ciel et terre" - sans aucune ponctuation - (BNT) 
II y a un véritable travail d'exégèse à la base de chaque texte. Chaque verset a été discuté, révisé, approuvé, dans un va-et-vient continuel entre les membres de chaque binôme et, à la fin, avec le comité éditorial. C'est vraiment un texte commun qui est offert. On sait que certains textes feront réagir, déstabiliseront parfois. II y a certains passages auxquels nous sommes plus habitués qui surprennent, comme les Béatitudes, par exemple. Pourtant, c'est parlant, vivant, actuel. Non pas pour ramener qui que ce soit dans un quelconque giron religieux, mais parce que ce sont des textes qui nous renvoient à nos conceptions du monde, à notre héritage, à nos questions de vie et de relations avec le réel et l'au-delà du réel.

\section{- Cela a-t-il fait l'objet d'un débat entre les exégètes?}

- On n'a pas eu encore beaucoup de réactions à ce niveau, mais des recensions devraient paraître bientôt. À la Faculté de théologie, lorsqu'on a présenté Genèse 1 à un groupe de professeurs retraités, la réaction a été : "Si on perd nos repères théologiques associés à un certain langage, qu'est ce qu'on fait?" Des questions de langage et de traditions sont posées. Comment réagir? En réinterprétant, en faisant surgir du neuf, en construisant, sans se séparer d'une tradition, mais sans rester prisonniers d'un univers traditionnel.

\section{- Vous avez le vertige parfois?}

- Oui, mais c'est extrêmement stimulant. Avoir proposé une traduction traditionnelle aurait été, à mes yeux, un travail inutile. Chez les exégètes plus que chez les écrivains, il y avait une volonté de changement, de remise en question des acquis trop ancrés dans les habitudes de lecture. Un exemple significatif : sans consigne initiale, presque tous les auteurs ont traduit ruah et $\pi v \varepsilon v \mu \alpha$ par "souffle" plutôt que par "esprit». Entre "le souffle de Dieu» et "l'esprit de Dieu", anthropologiquement parlant, on change de registre. Je disais, en plaisantant : "On peut avoir du monde sans esprit, mais pas du monde sans souffle". Avec un simple mot, on ouvre de nouveaux possibles, on invite à voir autrement. J'ai moi-même traduit, dans Josué, le mot torah par "enseignement" plutôt que par "loi»: "l'enseignement de Moise», non «la loi de Moise». Là aussi, on vient de changer de registre. Cela a un impact sur le genre de théologie que l'on veut faire.

II y a des choses que j'aurais aimé voir dans le texte mais qu'on 
n'a pas réussi à faire passer. Je pense en particulier à l'importance du langage inclusif. On a senti les réticences, parfois des résistances. Sur l'ensemble cependant, on voit les ouvertures que cette nouvelle traduction permet, parce qu'il y a une libération de la parole qui ne reste plus enfermée dans un carcan, mais qui oblige à questionner, à revoir ses propres catégories.

\section{- Comment la hiérarchie catholique a-t-elle pris votre travail?}

- La hiérarchie n'a pas été impliquée dans ce travail.

\section{- À aucune étape?}

- À aucune étape. Même si Bayard et Médiaspaul sont des maisons d'édition religieuses, chacun des binômes a été complètement libre de faire professionnellement son travail, respectueux du texte d'origine. La traduction finale a été présentée à la Commission doctrinale des évêques de France. Vous avez probablement vu la petite notice au début de la Bible. II n'y a pas d'«imprimatur»11 ce qui, pour moi, est une bonne chose. Un «imprimatur» aurait signifié que les catholiques s'appropriaient le texte. Par ailleurs, il y a dans la notice cette phrase : la commission «en encourage la lecture", réservant son jugement sur la foi de la réception de cette version par les catholiques. Bel exemple de l'importance accordée au sensus fidelium.

Le projet ne visait pas à aller chercher un «imprimatur» ou un nihil obstat. On voulait redonner ce texte à tout homme et toute femme, indépendamment de son appartenance religieuse ou non. On voulait que ce texte retrouve sa place dans la culture contemporaine. Les deux maisons d'édition religieuses, selon les lois du droit canon, ont demandé l'autorisation. Celle-ci leur a été donnée. Chez les évêques québécois et canadiens, il y a eu un accueil favorable accompagné d'une réserve parce que les évêques s'étaient fait échauder par une autre traduction à laquelle ils avaient donné un «imprimatur» un peu rapidement. Le principal, pour l'équipe, c'était que ces textes s'inscrivent dans la mouvance d'une littérature contemporaine.

\section{- Les gens ne viennent plus à l'église, vous, vous sortez dans la rue.}

- On ne désire pas aller chercher le monde, ni dans l'église, ni dans la rue. Mais on s'est dit qu'il fallait que ces textes restent un héritage

11. Traditionnellement, les livres religieux étaient cautionnés par un évêque qui ordonnait : «imprimatur» : que cela soit imprimé. 
fondamental pour la littérature occidentale contemporaine. Ils devaient donc être remis dans la rue, puisque c'est là qu'il faut qu'ils se retrouvent en bonne partie. Si les communautés chrétiennes se reconnaissent dans cette approche neuve des textes bibliques, nous ne pouvons qu'accueillir positivement leur choix et les encourager à poursuivre leurs découvertes de l'univers biblique à travers l'originalité de cette traduction. N'oublions pas que cela reste un texte religieux au départ. Quand on voit par ailleurs des expériences comme celles qui se font au Café Chaos, un café branché de Montréal, où chaque mois on lit un extrait des textes de la $B N T$, on ne peut que se réjouir d'être aussi dans la rue.

Il y a une écoute du texte parce qu'il y a une beauté du texte qui vient aussi susciter un questionnement. Si c'est dans la rue que cela se produit, parce que cela ne se fait plus dans les églises, tant mieux. Mais il n'est pas question de promouvoir le prosélytisme.

\section{- Vous vous situez dans le courant de la postmodernité. Qu'est- ce que la postmodernité pour un exégète?}

- Je ne sais pas ce qu'est «la» postmodernité et, par les temps qui courent, je défie quiconque d'en donner une seule définition englobant toutes les tendances actuelles. Ceci dit, pour moi, la postmodernité, ce n'est pas la négation de la modernité. Je ne me situe pas à ce niveau-là. Le courant dans lequel je m'inscris est celui d'une philosophie et d'une théologie de type processuelle (process theology) autour des travaux d'A.N. Whitehead pour la philosophie et la métaphysique, et de C. Hartshorne et J. Cobb (entre autres) pour ce qui touche les questions théologiques.

Je me situe dans un courant théologique et exégétique qui ne cherche pas à nier ce que nous a apporté la modernité, mais qui accorde aussi une place importante à l'aspect religieux de la réalité. On a eu tendance, au cours des derniers siècles, à créer une division opaque entre science et foi, à segmenter le réel. Je veux essayer de briser ce bris, de rompre cette rupture. Dans l'approche processuelle j'ai trouvé un lieu de réflexion qui me convient et qui me permet, comme exégète, de regarder le texte biblique en le considérant comme un discours possible, qui a sa place "dans" et "avec" un monde scientifique. Cette approche veut se situer en continuité avec l'héritage, tout en allant plus loin que ce que la modernité nous a apporté. 


\section{- Est-ce que cela modifie votre lecture de la Bible?}

- Comme historien ou archéologue, non. Mais quand je me situe comme exégète, quand je veux faire le travail d'interprétation du texte dans une herméneutique pour aujourd'hui, cela a un impact dans mon rapport au texte, dans ce que je vais m'attendre à trouver dans les textes, et aussi dans la façon dont je vais reprendre le texte pour le faire réatterrir dans le monde contemporain. Cela m'oblige à poser aux textes des questions différentes de celles auxquelles s'en tiennent les discours religieux traditionnels.

Par exemple, on a souvent considéré Dieu, en théologie traditionnelle, comme unique créateur, omnipotent, omniscient. Dans une perspective processuelle, on va qualifier ces concepts d'omnipotence et d'omniscience, leur fournir un cadre d'application qui soit compatible avec les perspectives cosmologiques contemporaines de devenir ouvert et de créativité permanente. Dieu, dans ce contexte, n'est plus seulement le transcendant et le lointain. On sera davantage porté à considérer l'aspect contingent du Dieu-avec, qui chemine avec et dans le devenir. Je vais être ainsi plus à l'affût de ces réalités suggérées aussi dans les textes bibliques. Ce regard porté sur les textes nous invite à prendre en considération davantage de facettes de Dieu que ce à quoi la tradition et la doctrine nous ont habitués (ou confinés).

\section{- Vous considérez Dieu tel qu'il nous apparaît dans ces textes là?}

- Certainement pas. II est normal que nous devions employer des métaphores pour tenter de dire l'entité divine. Le problème, c'est que l'on a privilégié certaines métaphores au détriment d'autres qui ont aussi un certain pouvoir d'évocation. Prenons un exemple. Quand je lis un texte comme Genèse 22, 12 : "Arrête, car maintenant je sais que tu crains Dieu", le texte laisse entendre qu'avant, Dieu ne savait pas. Que fais-je avec un texte semblable, qu'est-ce que j'en dégage? Pour une certaine théologie, il n'est pas possible que Dieu ne sache pas : Dieu sait tout! On nous a appris qu'il était omniscient. Mais j'ai un texte qui semble proposer autre chose ! Si je prends cette métaphore aussi au sérieux que celles parlant d'un Dieu qui sait tout, je me trouve devant quelque chose qui questionne certaines images de Dieu. Qu'est-ce que je fais d'un texte comme celui-là? Quelle théologie puis-je en dégager? Le classer purement et simplement dans la catégorie des anthropomorphismes à bannir? Dire que ce 
n'est pas vraiment ce que l'auteur voulait suggérer? Ou penser une théologie dans laquelle le Dieu-avec est aussi le Dieu qui apprend et, donc, qui devient? N'est-ce pas d'ailleurs la traduction même du nom YHWH : 'eyeh asher 'eyeh "je deviens qui je deviens" (Ex 3,14), forme verbale inaccomplie en hébreu!

Autre exemple : "Je me repens du mal que j'ai voulu faire». Que peut-on construire et proposer à partir de cette image d'un Dieu qui se repent? Comment rendre compte de ces textes pour qu'ils soient théologiquement signifiants? "Dieu vit que cela était bon" dit le premier récit de la Genèse, et non «Dieu savait d'avance que cela allait être bon". Encore une fois, une prise au sérieux de cette proposition oblige à porter un regard neuf, différent, sur certaines images de Dieu que l'on a transmises dans la tradition. Le texte m'oblige à poser des questions neuves et mes questions, à leur tour, me font voir le texte sous des aspects souvent insoupçonnés. L'approche de type process n'est pas une panacée. Elle a cependant l'avantage de tenter de tenir compte de toutes les possibilités, même celles (et peut-être surtout celles) qui demandent de créer du neuf sur les bases de l'ancien.

\section{- Le concept de Dieu a évolué...}

- Bien sûr que le concept de Dieu a évolué. J'irais jusqu'à dire que Dieu évolue. Une part importante des concepts théologiques chrétiens sont issus des IVe, Ve siècles, alors que l'on avait une vision du monde que l'on considérait comme fermé, statique, fini. On n'a plus le même rapport au cosmos et à Dieu, mais notre langage reste associé à des images des IV et $\mathrm{Ve}$ siècles. Pourtant, tout autour de nous nous invite à considérer l'univers comme ouvert, dynamique, en devenir permanent. Peut on faire entrer en dialogue les textes et la nouvelle vision du monde? C'est le défi que la postmodernité me lance. Plus je fréquente les textes anciens et plus je découvre qu'ils sont loin d'être démodés. Une condition cependant doit être respectée pour qu'un réel dialogue s'instaure entre ces textes anciens et notre monde postmoderne : que notre approche ne soit pas basée sur "quelle réponse le texte va m'apporter", mais plutôt sur "quelle(s) question(s) me pose-t-il?».

\section{- C'est un défi...}

- C'est un énorme défi. C'est un nouveau chantier, complètement ouvert. Si on veut avoir une pertinence pour aujourd'hui, si on 
veut que ces textes restent parlants pour aujourd'hui, il faut accepter l'invitation d'inventer, de faire du neuf, de sortir des sentiers battus. II faut avoir le courage de relever ce défi, de se laisser questionner par les textes et tenter de trouver quelques pistes de réponses pour nous, aujourd'hui. Si réponse il y a...

C'est un défi de trouver un langage pertinent dans un monde qui innove, et de l'inscrire dans une tradition qui, par définition, a tendance à être plutôt conservatrice. C'est en ce sens que je suis en postmodernité. Je pense que le discours religieux a encore toute sa place. II faut toutefois être en mesure de lui donner la portée et le langage adaptés à la réalité d'aujourd'hui. Ceci dit, il ne s'agit pas de se mettre à la mode du jour et de balancer par-dessus bord tout l'héritage que nous portons. II faut plutôt trouver ce difficile équilibre entre tradition et nouveauté, héritage et avancée créatrice.

\section{- Qu'est ce qu'être croyant ou chrétien pour vous?}

- C'est nécessairement questionner, chercher, avancer.

\section{- C'est avoir un certain dogme aussi?}

- C'est m'inscrire dans la mouvance d'une communauté qui cherche, qu'on la qualifie de chrétienne, catholique, croyante (ce ne sont que des catégories larges ou minces). Qu'est-ce qu'être un croyant pour moi aujourd'hui? C'est considérer l'entité divine comme partie prenante d'un processus. II y a un faire advenir un monde ensemble, dans lequel l'entité divine a une place. Cela ne se vit pas seul, cela se vit avec d'autres, dans une communauté plus ou moins élargie et signifiante. Pour moi être croyant, ce n'est pas une expérience individuelle, c'est une expérience communautaire. Je ne crois pas pour moi, je crois avec d'autres et pour d'autres.

\section{- Quel rapport quelqu'un qui n'a pas la fol peut-il avoir avec ces textes-là?}

- Quelqu'un qui n'a pas la foi? Je ne sais pas si l'on a la foi, mais c'est là un langage commode. Je ne suis pas gêné de dire à quelqu'un qui lit ces textes : on va regarder ce qu'il y a dedans en étant attentif aux expériences humaines, aux rapports des humains entre eux, l'entité divine et le cosmos. Je parlerais presque d'une éthique, d'une éthique simplement proposée. II s'agit de propositions 
à soupeser et à faire sienne. J'aime bien ce texte du Deutéronome : "Vois, je te propose aujourd'hui le choix entre vie et bonheur, mort et malheur... Choisis donc la vie» (Dt 30,15.20). C'est pour cela que je préfère la théologie du pédagogue à celle du juriste, celle de la voie plutôt que celle de la loi. Le discours théologique doit partir non pas des concepts, mais de la vie. Je peux lire ces textes avec des gens qui ont simplement une expérience humaine, parce que c'est aussi de cela qu' ils parlent.

Les textes bibliques sont d'abord une expérience humaine qu'on lit à la lumière du rapport qu'on a au divin. Si quelqu'un me dit : "Je n'ai pas de rapport au divin, mais j'ai un rapport aux humains", je pense qu'on a là une multitude d'expériences humaines qui sont celles d'hommes et de femmes en recherche, avec les hauts et les bas, les bons coups et les échecs. C'est une recherche que l'on a souvent coiffée du nom de Yahvé, de Dieu, d'Élohim, etc., mais, fondamentalement, c'est d'abord une expérience humaine. Je peux regarder, dans le texte, les cris du cœur qui sont ceux de gens qui souffrent. Je lis dans les textes la volonté d'avoir une terre, d'avoir une place, d'avoir une stabilité, d'avoir une famille, d'être reconnu. $\mathrm{Ce}$ sont ces expériences que l'on rencontre dans les textes, rien d'autre.

\section{- Qu'est-ce qu'un rapport au divin?}

- C'est ce qui donne une direction. Mon rapport au divin, c'est l'expérience d'une avancée créatrice. C'est l'expérience du "faire advenir vers un plus". Quand je lis le texte biblique, c'est là que je me situe. C'est un sentir (feeling) selon l'expression de Whitehead, un sentir que le réel est plus que la somme des éléments mis les uns à côté des autres, un sentir du rapport à un surplus versus un nivellement par le bas. Je ne peux pas le prouver, je ne peux pas le démontrer : on ne serait plus dans le domaine de la foi, on serait dans celui de la raison. Cela n'est peut-être pas raisonnable, mais c'est raisonné. Le rapport au divin, c'est quelque chose d'existentiel, ce n'est pas quelque chose de cérébral, c'est un sentir indéfinissable mais réel d'une entité qui est là, au cœur même de tous les devenirs.

\section{- Le divin, est-ce une force, est-ce une âme?}

- Je ne suis pas capable de le définir, mais je lui donne des attributs. On a encore la perspective du divin comme étant atemporel. Je ne peux plus fonctionner avec des catégories semblables. 
L'anglais a de belles expressions, difficilement traduisibles en français. On considère souvent l'entité divine comme timeless, alors que je préfère en parler comme d'une entité timefull. On la considère comme spaceless, alors qu'on peut la considérer comme spacefull, omni-spatial plutôt que sans espace, réservoir du temps mais, en même temps, se temporalisant. C'est dans ces catégories-là, entre autres, que se joue ma relation processuelle au divin.

L'entité divine, c'est ce qui pousse en avant, ce qui offre la créativité plutôt que la répétition, ce qui invite à faire advenir le neuf plutôt qu'à reproduire. C'est d'abord et avant tout un être-avec. C'est très paulinien comme expression, même si je ne me sens pas très paulinien habituellement, mais c'est parlant : être-avec, pas seulement être avec l'humain, mais être-avec l'ensemble de ce qui existe, y compris l'être humain.

\section{- C'est tout autre chose que l'archéologie!}

- Tout à fait. Ce qui m'intéresse comme exégète du XXIe siècle, ce sont les propositions d'hommes et de femmes qui ont posé les questions de sens en relation avec le divin. Le point de vue de l'herméneutique, c'est celui d'une rencontre possible non seulement avec l'archéologie du texte, mais surtout avec un sentir qui nous est proposé dans le texte et qui nous est renvoyé aujourd'hui pour que nous lui donnions sens.

\section{Maurice Burgevin}

